\title{
Prevention of obesity in mice by antisense oligonucleotide inhibitors of stearoyl-CoA desaturase-1
}

\author{
Guoqiang Jiang, ${ }^{1}$ Zhihua Li, ${ }^{1}$ Franklin Liu, ${ }^{1}$ Kenneth Ellsworth, ${ }^{1}$ Qing Dallas-Yang, ${ }^{1}$ \\ Margaret Wu, ${ }^{1}$ John Ronan, ${ }^{2}$ Christine Esau, ${ }^{3}$ Cain Murphy, ${ }^{3}$ Deborah Szalkowski, ${ }^{1}$ \\ Raynald Bergeron, ${ }^{1}$ Thomas Doebber, ${ }^{1}$ and Bei B. Zhang ${ }^{1}$ \\ ${ }^{1}$ Metabolic Disorders — Diabetes and ${ }^{2}$ Comparative Medicine, Merck Research Laboratories, Rahway, New Jersey, USA. \\ ${ }^{3}$ Isis Pharmaceuticals, Carlsbad, California, USA.
}

\begin{abstract}
Effective therapies for the treatment of obesity, a key element of metabolic syndrome, are urgently needed but currently lacking. Stearoyl-CoA desaturase-1 (SCD1) is the rate-limiting enzyme catalyzing the conversion of saturated long-chain fatty acids into monounsaturated fatty acids, which are major components of triglycerides. In the current study, we tested the efficacy of pharmacological inhibition of SCD1 in controlling lipogenesis and body weight in mice. SCD1-specific antisense oligonucleotide inhibitors (ASOs) reduced SCD1 expression, reduced fatty acid synthesis and secretion, and increased fatty acid oxidization in primary mouse hepatocytes. Treatment of mice with SCD1 ASOs resulted in prevention of diet-induced obesity with concomitant reductions in SCD1 expression and the ratio of oleate to stearoyl-CoA in tissues and plasma. These changes correlated with reduced body adiposity, hepatomegaly and steatosis, and postprandial plasma insulin and glucose levels. Furthermore, SCD1 ASOs reduced de novo fatty acid synthesis, decreased expression of lipogenic genes, and increased expression of genes promoting energy expenditure in liver and adipose tissues. Thus, SCD1 inhibition represents a new target for the treatment of obesity and related metabolic disorders.
\end{abstract}

\section{Introduction}

Metabolic syndrome has become one of the leading health problems in the world, particularly in developed countries. As a component of metabolic syndrome, obesity also has causal roles in other components of the syndrome, including insulin resistance, dyslipidemia, and cardiovascular diseases. Effective treatments for metabolic syndrome in general and obesity in particular have been lacking $(1,2)$.

Stearoyl-CoA desaturases (SCDs) convert saturated long-chain fatty acids into monounsaturated fatty acids (MUFAs) and are the rate-limiting enzymes in the biosynthesis of MUFAs in vivo. The preferred substrates are palmitoyl-CoA (16:0) and stearoyl-CoA (18:0), which are converted into palmitoleoyl-CoA (16:1) and oleoylCoA (18:1), respectively. The resulting MUFAs are major components of triglycerides, cholesterol esters, and phospholipids (3).

The mouse has $4 S C D$ gene isoforms (SCD1-4). SCD1 is expressed in a broad range of tissues and at high levels in the insulin-responsive liver, white adipose tissue (WAT), and brown adipose tissue (BAT). On the other hand, $S C D 2, S C D 3$, and $S C D 4$ are expressed predominantly in the brain, the Harderian gland, and the heart, respectively (4-6). There are 2 known human $S C D$ isoforms that show approximately $85 \%$ homology to murine $\operatorname{SCD} 1(7,8)$. The

Nonstandard abbreviations used: ACC1, acetyl-coenzyme A carboxylase 1; ACO, acyl-CoA oxidase; ASO, antisense oligonucleotide; ASOctrl, control ASO; $\beta 3$-AR, $\beta 3$-adrenergic receptor; BAT, brown adipose tissue; FAS, fatty acid synthase; FIAF, fasting-induced adipocyte factor; HFD, high-fat diet; L-CPT1, liver-specific carnitine palmitoyltransferase $1 ; \mathrm{mpk}$, mg per kg body weight; MUFA, monounsaturated fatty acid; PE, petroleum ether; SCD, stearoyl-CoA desaturase; UCP, uncoupling protein; VLCAD, very-long-chain acyl-CoA dehydrogenase; WAT, white adipose tissue. Conflict of interest: G. Jiang, Z. Li, F. Liu, K. Ellsworth, Q. Dallas-Yang, M. Wu, J. Ronan, D. Szalkowski, R. Bergeron, T. Doebber, and B.B. Zhang potentially own stock and/or hold stock options in Merck \& Co., Inc.

Citation for this article: J. Clin. Invest. 115:1030-1038 (2005). doi:10.1172/JCI200523962. expression of $S C D$ isoforms is highly regulated by multiple factors, including dietary factors and hormones $(4,9-11)$.

Emerging evidence suggests that SCD1 plays a crucial role in lipid metabolism and body weight control $(12,13)$. Asebia mice are homozygous for a naturally occurring mutation that results in the lack of SCD1 expression (14). The asebia mice manifest defective hepatic cholesterol ester and triglyceride synthesis (15), are lean and hypermetabolic, and have reduced liver steatosis (16). Similar phenotypes were reported for $S C D 1$-knockout mice, which are also resistant to diet-induced insulin resistance (17). Finally, SCD1 deficiency also reduces hepatic steatosis in lipodystrophic aP2-nSREBP-1c mice, which express a constitutively active form of the SREBP-1c (18).

The detailed mechanisms by which SCD1 deficiency affects body weight and adiposity are not completely understood. Leptin may exert its metabolic effects by inhibiting SCD1 (16-19). SCD1knockout mice have increased activity of AMP-activated protein kinase, which may be responsible for the regulation of some of the genes important in lipid metabolism (19). Most recently, it was shown that $S C D 1$ deficiency increases basal expression of uncoupling proteins (UCPs) $1-3$ and $\beta 3$-adrenergic receptors ( $\beta 3$-ARs) in BAT and increases basal thermogenesis in mice (20).

The above-mentioned studies suggest that SCD1 deficiency reduces body weight and adiposity by increasing basal metabolism in mice. It was also reported that higher SCD activity as indicated by higher "desaturation index" (the ratio of oleate to stearoyl-CoA or 18:1/18:0) is strongly correlated with higher plasma triglyceride levels in humans (21). It thus appears that inhibition of SCD1 may represent a novel approach for the treatment of metabolic syndromes in human subjects (12). However, interpretation of results from studies on genetic models of SCD1 deficiency was complicated, since SCD1-deficient mice develop alopecia, or hair loss (14). It remains a possibility that increased heat dissipation via 

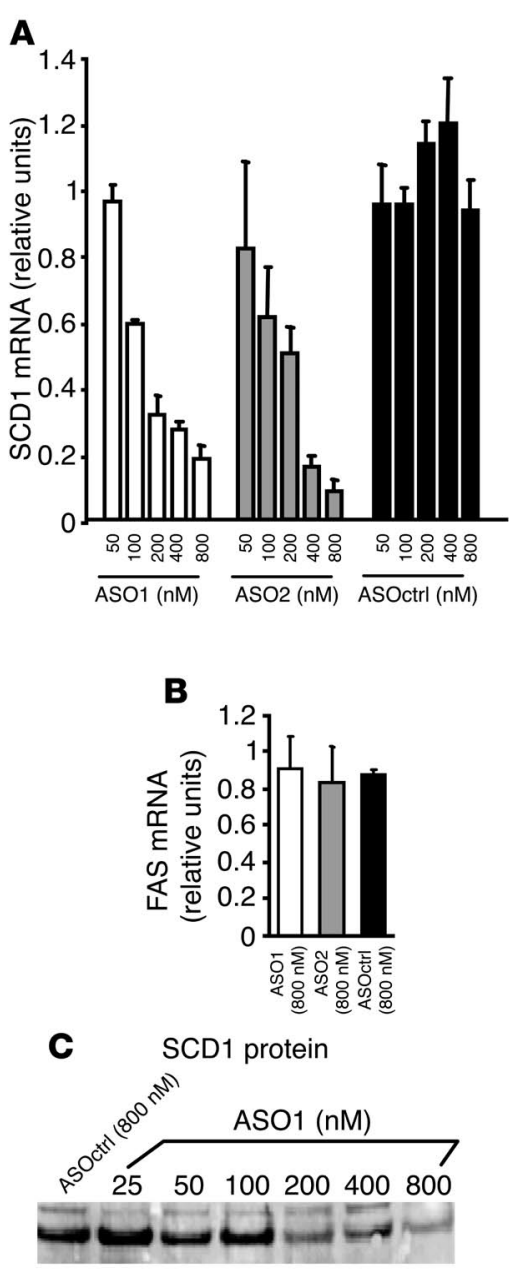
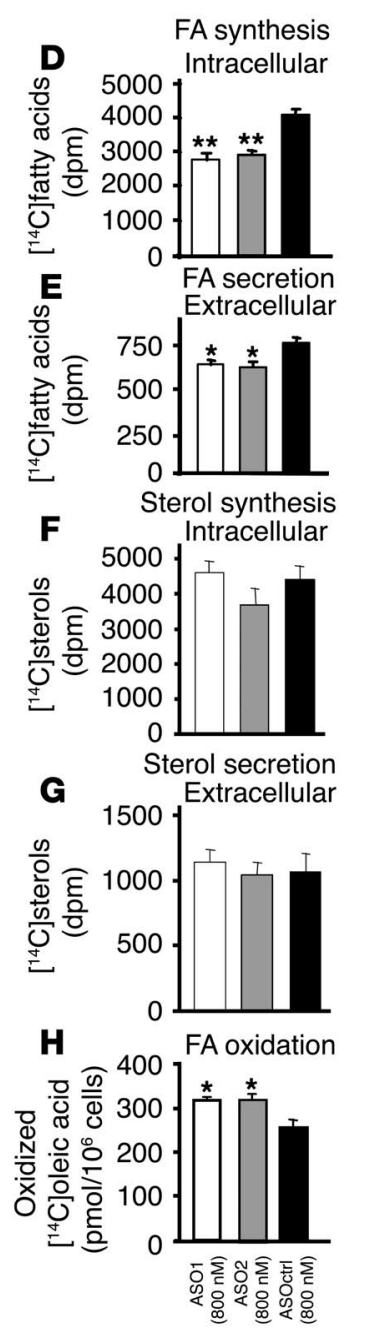

the abnormal skin may at least partially be responsible for reduced body weight and adiposity. Additionally, SCD1 deficiency has been shown to improve insulin sensitivity in SCD1-knockout mice but not in the aP2-nSREBP-1c mice $(17,18,22)$. The role of SCD1 in insulin sensitivity therefore remains unclear. Finally, genetic models do not allow the question of whether pharmacological inhibition of SCD1 can also improve metabolic regulation to be addressed.

The goal of the current study, which uses antisense oligonucleotide (ASO) as a treatment to inhibit SCD1 expression and activity in vivo, was to investigate the effects of pharmacological SCD1 inhibition on metabolic regulation. The results show that SCD1 ASOs reduce $S C D 1$ expression in vitro and in vivo prevent diet-induced obesity in animals in the absence of alopecia and improve insulin sensitivity in the mice on a high-fat diet (HFD). Thus, the study demonstrates that SCD1 inhibitors represent a new therapeutic approach for the treatment of obesity and metabolic syndrome.

\section{Results}

SCD1 ASOs decreased SCD1 mRNA and protein levels in cultured primary mouse hepatocytes. Primary hepatocytes isolated and cultured from C57/B6 mice were transfected with varying concentrations (50-800 nM) of SCD1-specific ASOs (ASO1 and ASO2) and a scrambled control ASO (ASOctrl). After the mice recovered overnight, RNA and proteins were extracted from the transfected cells.

\section{Figure 1}

Effects of SCD1 ASOs on SCD1 mRNA and protein levels, fatty acid and sterol synthesis and secretion, and fatty acid oxidation in primary mouse hepatocytes in vitro. Primary hepatocytes isolated from C57/B6 mice were transfected with ASO1, ASO2 and ASOctrl. Shown are the relative abundances of SCD1 (A) and FAS (B) mRNA levels in the transfected hepatocytes as quantified by TaqMan real-time PCR. SCD1 protein levels were determined by Western blot analysis (C). Also shown are the levels of conversion of $\left[{ }^{14} \mathrm{C}\right]$ acetate into $\left[{ }^{14} \mathrm{C}\right]$-labeled fatty acids in hepatocytes (intracellular; D), $\left[{ }^{14} \mathrm{C}\right]$-labeled fatty acids secreted into the tissue culture medium (extracellular; $\mathbf{E}),\left[{ }^{14} \mathrm{C}\right]$ sterols in hepatocytes (intracellular; $\mathbf{F}$ ), $\left[{ }^{14} \mathrm{C}\right]$ sterols in the culture medium (extracellular; $\mathbf{G}$ ), and conversion of $\left[{ }^{14} \mathrm{C}\right]$ oleic acid into soluble $\left[{ }^{14} \mathrm{C}\right]$-labeled products in the cells $(\mathbf{H})$. The experiments were repeated 3 times with similar results.

The RNA was used to prepare cDNA by reverse transcription. Realtime quantitative RT-PCR (TaqMan) analysis showed that the levels of SCD1 mRNA were reduced by both ASO1 and ASO2 but not ASOctrl in a dose-dependent fashion, with an $\mathrm{IC}_{50}$ of approximately $150 \mathrm{nM}$ (Figure 1A). The levels of fatty acid synthase (FAS), which was used as a control, were not affected by any of the ASOs in the treatment time frame (Figure 1B). Western blot analysis of protein extracts from the hepatocytes showed that both ASO1 and ASO2 but not ASOctrl reduced SCD1 protein levels in the primary hepatocytes in a dose-dependent fashion (Figure 1C and data not shown). Taken together, these results clearly demonstrated that ASO1 and ASO2 reduce SCD1 mRNA and protein levels in a specific and dose-dependent fashion in primary hepatocytes.

SCD1 ASOs inbibited fatty acid synthesis and secretion and increased fatty acid oxidation in primary mouse hepatocytes. After overnight culture, primary hepatocytes transfected with 800 nM ASO1, ASO2, or ASOctrl were incubated with medium containing either $\left[{ }^{14} \mathrm{C}\right]$ acetate or $\left[{ }^{14} \mathrm{C}\right]$ oleic acid for another 17 hours. For cells incubated with $\left[{ }^{14} \mathrm{C}\right]$ acetate, the levels of $\left[{ }^{14} \mathrm{C}\right]$-labeled fatty acids and sterols in both the cells and the culture medium were then quantified at the end of the incubation. The results showed that, in comparison with cells transfected with ASOctrl, cells transfected with either ASO1 or ASO2 had significantly reduced levels of $\left[{ }^{14} \mathrm{C}\right]$-labeled fatty acids both in the hepatocytes (Figure 1D) and in the culture medium (Figure 1E). On the other hand, the levels of $\left[{ }^{14} \mathrm{C}\right]$ sterols in the cells or in the culture medium transfected with all of the 3 ASOs were comparable (Figure 1, F and G).

For cells incubated with $\left[{ }^{14} \mathrm{C}\right]$ oleic acids, the levels of soluble $\left[{ }^{14} \mathrm{C}\right]-$ labeled products in the tissue culture medium were quantified at the end of the incubation. In the culture media of cells transfected with either ASO1 or ASO2, higher levels of soluble $\left[{ }^{14} \mathrm{C}\right]$-labeled products were converted from $\left[{ }^{14} \mathrm{C}\right]$ oleic acid (Figure $1 \mathrm{H}$ ). Taken together, these results showed that reduction of SCD1 by ASO1 and ASO2 decreased fatty acid synthesis and secretion while increasing fatty acid oxidation in primary hepatocytes in vivo.

SCD1 ASOs reduced weight gain, body adiposity, and plasma insulin and glucose levels. C57/B6 mice were switched from regular diet to HFD and treated with ASO1, ASO2, and ASOctrl at 5 and $15 \mathrm{mg}$ per kg body weight (mpk) twice a week for 10 weeks. In comparison with mice treated with ASOctrl, those treated with ASO1 and ASO 2 at $15 \mathrm{mpk}$ but not $5 \mathrm{mpk}$ showed significantly reduced body weight gain $(P<0.05)$ starting at week 4 of the experiment (Figure 2A). As shown in Figure 2B, in the first 2-3 weeks, food intake went down substantially for mice in all treatment groups as they started to feed on the more energy-rich HFD. Through- 

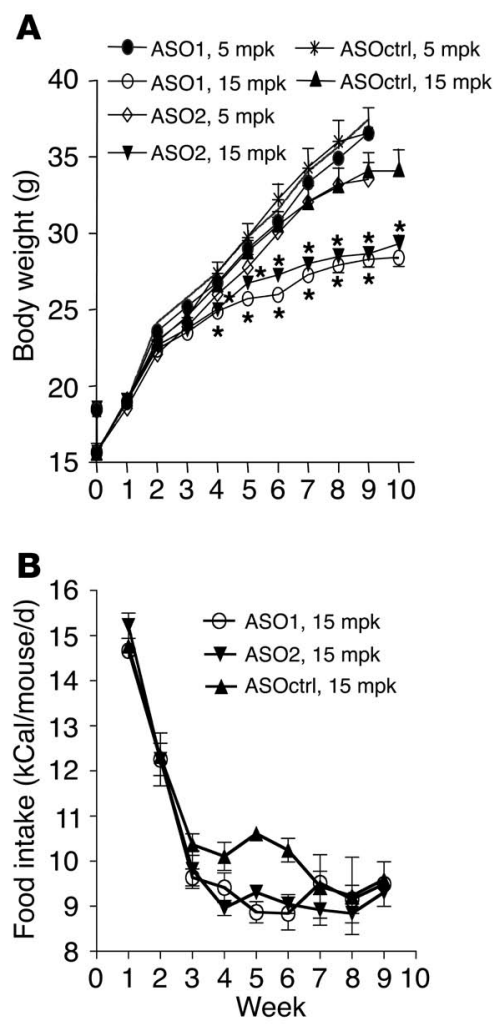

C
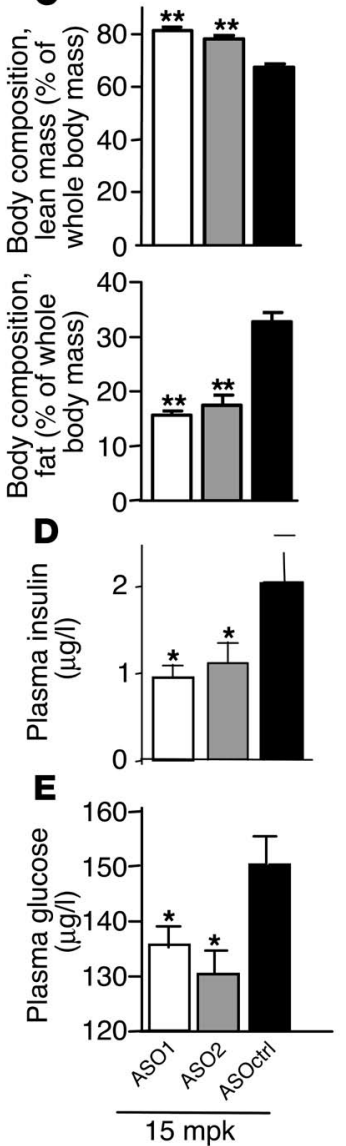

out the course of the experiment, however, ASO treatments did not appear to significantly affect food intake, which suggests that body weight reduction in the SCD1 ASO-treated groups was not due to decreased food intake (Figure 2B). Body composition analysis by nuclear magnetic resonance showed that, in comparison with mice treated with ASOctrl at similar dosage, mice treated with ASO1 and ASO2 at 15 but not $5 \mathrm{mpk}$ had a significantly reduced percentage of fat but an increased percentage of lean mass to total body mass $(P<0.05$; Figure $2 \mathrm{C}$ and data not shown). These results suggest that, without affecting food intake, SCD1 ASOs protected the mice from HFD-induced obesity in a dose-dependent fashion. Furthermore, mice treated with either ASO1 or ASO2 at $15 \mathrm{mpk}$ exhibited significantly lower postprandial plasma glucose and insulin concentrations (Figure 2, E and D), which suggests that the SCD1 ASO-treated mice have improved insulin sensitivity. On the other hand, ASO treatments

\section{Figure 3}

Effect of SCD1 ASO2 on oxygen consumption and physical activity of C57/B6 mice on HFD. Interval measurements of oxygen consumption and physical activity were done every 30 minutes for 24 hours on C57/B6 mice fed HFD and treated with ASO2 or ASOctrl at $15 \mathrm{mpk}$ for 10 weeks as described in Methods. Shown are the volume of oxygen consumed (A) and the cumulative ambulatory $x y$ counts (i.e., the number of times that light beams in the $x$ and $y$ axes were broken due to animal movement; B). Both oxygen consumption and physical activity are significantly higher in ASO2-treated mice than in ASOctrl-treated mice $(P<0.05)$.

\section{Figure 2}

Effect of SCD1 ASOs on weight gain, body adiposity, and plasma metabolic parameters in C57/B6 mice on HFD. C57/B6 mice were given HFD and treated with ASO1, ASO2, or ASOctrl at 5 and 15 mpk twice a week for 9-10 weeks as described in Methods. Shown are the body weight (A) and food intake (B) of the animals measured weekly. Also shown are body composition (C), and postprandial plasma insulin (D) and glucose (E) measured at the end of the treatments. While ASO2 was only tested in the current experiment, similar results on ASO1 and ASOctrl were obtained in 2 experiments. ${ }^{*} P<0.05$; ${ }^{* \star} P<0.01$.

did not significantly alter plasma free fatty acid and triglyceride concentrations in the mice (data not shown).

SCD1 ASO2 increased both metabolic rate and physical activity. The effect of SCD1 ASO treatment on both oxygen consumption, an indicator of energy expenditure, and physical activity of the mice was also investigated. The results showed that, over a period of approximately 21 hours, mice treated with ASO2 at $15 \mathrm{mpk}$ for 10 weeks had significantly higher rates of oxygen consumption (Figure $3 \mathrm{~A}$ ) as well as physical activity levels (Figure 3B) $(P<0.05)$. We also calculated resting energy expenditure by computing the oxygen consumption associated with the lowest $20 \%$ physical activity recordings $(n=$ about $9-10$ recordings out of a total of approximately 45-50 recorded intervals). This result showed that resting energy expenditure was also increased significantly, by $12 \%$, in the ASO2-treated animals $(2,426 \pm 53 \mathrm{ml} / \mathrm{kg} / \mathrm{h})$ as compared with the ASOctrl-treated ones $(2,131 \pm 76 \mathrm{ml} / \mathrm{kg} / \mathrm{h} ; P<0.01)$.

$S C D 1$ ASOs reduced SCD1 mRNA levels, enzyme activity, and lipid "saturation index" in a dose- and time-dependent fashion in liver. In comparison with ASOctrl at $15 \mathrm{mpk}$, ASO1 and ASO2 at $15 \mathrm{mpk}$ reduced both hepatic SCD1 mRNA (Table 2) and SCD1 activity (Figure 4A) by approximately $50 \%$ after 4 weeks of treatment. After 10 weeks of treatment, both ASO1 and ASO2 inhibited liver SCD1 dose dependently, with approximately $60 \%$ reduction in mRNA (Table 2 ) and

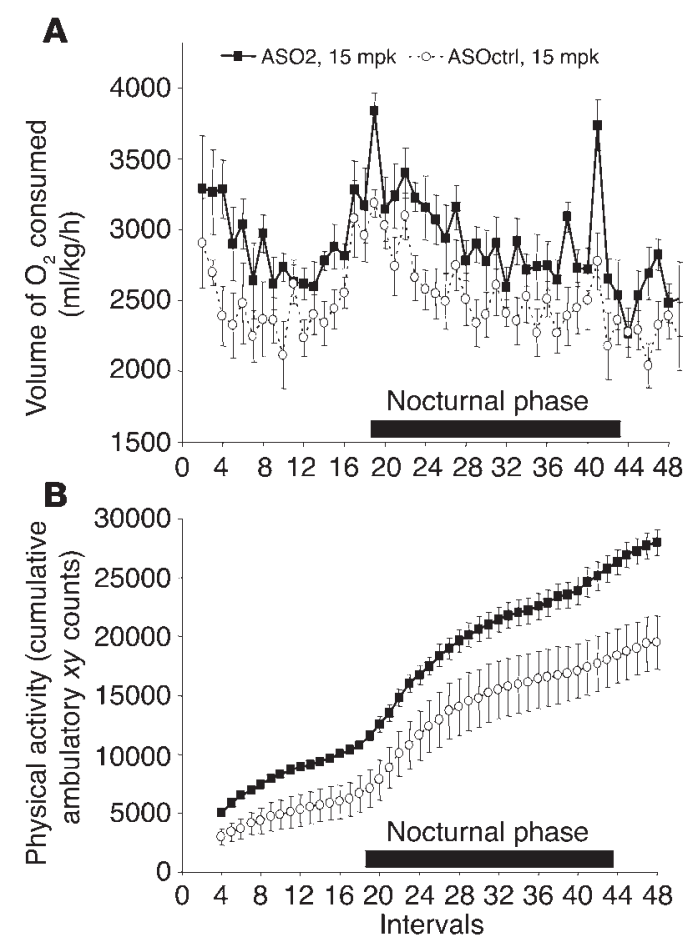


A
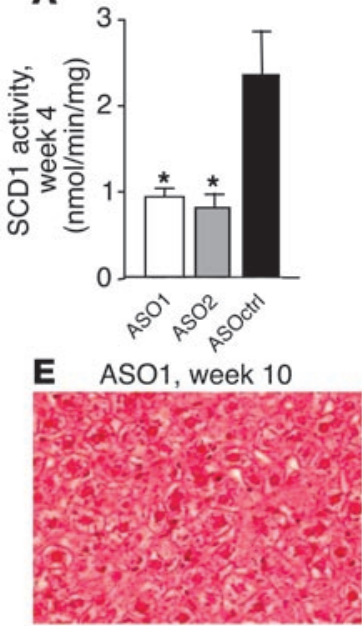

B

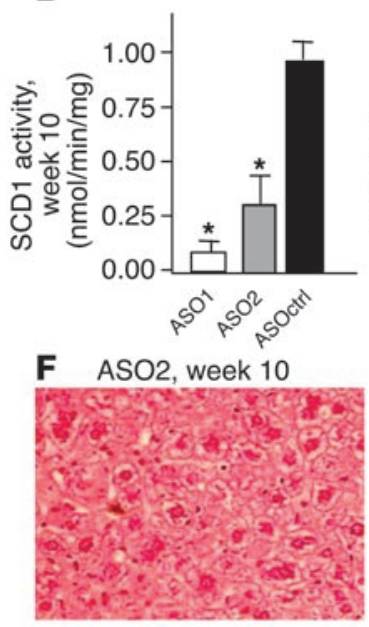

C

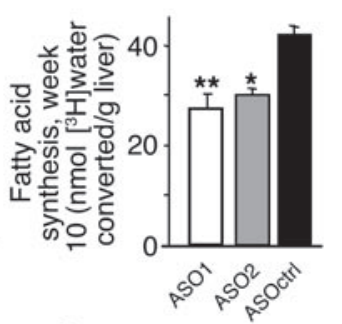

G ASOctrl, week 10

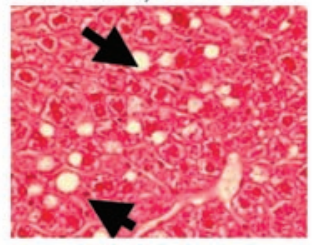

D

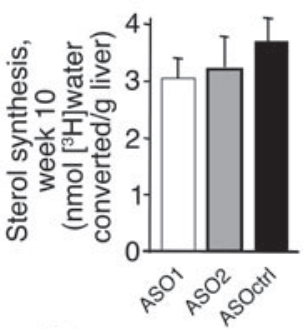

H ASOctrl, week 10

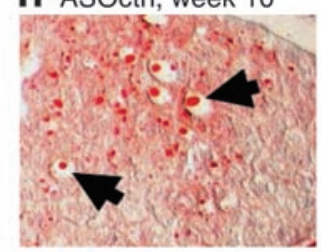

\section{Figure 4}

Effect of SCD1 ASOs on SCD1 enzymatic activity, de novo fatty acid synthesis activity, and fat accumulation in the liver of treated C57/B6 mice on HFD. SCD1 enzymatic activity in the liver of mice treated with the various ASOs at $15 \mathrm{mpk}$ for 4 weeks (A) and 10 weeks (B). The de novo fatty acid synthesis activity as indicated by the level of conversion of $\left[{ }^{3} \mathrm{H}\right]$ water into fatty acids (C) or sterols (D) in the liver tissues of mice treated with the various ASOs at 15 mpk for 10 weeks. (E-G) Images of H\&E staining of liver sections of mice treated with the ASO1 (E), ASO2 (F) and ASOctrl (G) at 15 mpk for 10 weeks. The clear vacuoles in the liver section are identified by arrows in $\mathbf{G}$. (H) Images of oil red $O$ staining of frozen liver section of a mouse treated with ASOctrl at $15 \mathrm{mpk}$ for 10 weeks. The lipid drops stained by oil red $\mathrm{O}$ within the vacuoles are identified by arrows.

approximately $65-90 \%$ reduction in enzyme activity (Figure $4 \mathrm{~B}$ ) at $15 \mathrm{mpk}$. Finally, treatment with ASO1 and ASO2 at $15 \mathrm{mpk}$ for 4 and 10 weeks significantly reduced the saturation index of C16 and C18 fatty acids in both liver and plasma (Figure 5 and data not shown).

Treatment with SCD1 ASOs altered the expression of multiple lipid metabolism genes in the liver. ASO1 and ASO2 at $15 \mathrm{mpk}$ did not significantly affect the mRNA levels of FAS, acetyl-coenzyme A carboxylase 1 (ACC1), and SREBP-1 in the liver after 4 weeks of treatment. After 10 weeks of treatment, however, FAS, ACC1, and SREBP-1 mRNA levels were reduced by both ASO1 and ASO2, mostly in a dose-dependent fashion (Table 2). A different temporal regulation was seen with the ACC2 gene, as ACC2 mRNA was already significantly reduced by approximately $60 \%$ by both ASO1 and ASO2 after 4 weeks of treatment and remained similarly reduced throughout the experiment (Table 2).

Both ASO1 and ASO1 significantly increased liver-specific carnitine palmitoyltransferase 1 (L-CPT1) mRNA levels after 4 weeks of treatment at $15 \mathrm{mpk}$ and after 10 weeks of treatment at 15 but not $5 \mathrm{mpk}$. The mRNA levels of several other genes measured as a control, including very-long-chain acyl-CoA debydrogenase $(V L C A D)$ and acyl-CoA oxidase (ACO), were not significantly different among animals treated with all 3 ASOs at all dosages after both 4 and 10 weeks of treatment (data not shown).

SCD1 ASOs reduced de novo fatty acid synthesis and steatosis in the liver. After 10 weeks of treatment, we determined the activity of de novo fatty acids and sterol synthesis in the livers of mice treated with various ASOs at $15 \mathrm{mpk}$ by measuring the levels of $\left[{ }^{3} \mathrm{H}\right]$-labeled fatty acids and sterols extracted from liver after i.p. injection of $\left[{ }^{3} \mathrm{H}\right]$ water. The results showed that ASO1 and ASO2 at $15 \mathrm{mpk}$ significantly reduced the levels of $\left[{ }^{3} \mathrm{H}\right]$-labeled fatty acids but not $\left[{ }^{3} \mathrm{H}\right]$-labeled sterols in the liver of the treated mice (Figure 4, C and D). $\mathrm{H} \& \mathrm{E}$ staining revealed vacuoles in the liver section from mice treated with ASOctrl (Figure 4G) but not with ASO1 (Figure 4E) or ASO2 (Figure 4F) at 15 mpk for 10 weeks. Such vacuoles are known to represent empty spaces typically left behind by lipid drops, which were dissolved during the H\&E procedure. Staining with lipid dye oil red $\mathrm{O}$ confirmed the presence of lipid drops within the vacuoles in the frozen sections of liver tissue from the ASOctrl-treated mice (Figure 4H). Taken together, these results demonstrated that ASO1 and ASO2 at 15 mpk reduced both de novo fatty acid synthesis activity and HFD-induced hepatic steatosis in treated mice.

$S C D 1$ ASOs reduced SCD1 and FAS mRNA levels in WAT. In comparison with ASOctrl, ASO2 at $15 \mathrm{mpk}$ reduced SCD1 mRNA levels by approximately $70 \%$ in WAT after either 4 or 10 weeks of treatment (Table 3). The ASO2 treatment also significantly reduced FAS mRNA levels (reduced about 70-75\%) in WAT after 10 but not 4 weeks of treatments (Table 3). The ASO2 treatment did not significantly affect the mRNA levels of $V L C A D$, which were measured as a control, at either week 4 or week 10 (data not shown).

$S C D 1$ ASOs reduced SCD1 $m R N A$ levels while increasing the $m R N A$ levels of multiple thermogenesis-related genes in BAT. In comparison with ASOctrl at $15 \mathrm{mpk}$, ASO 1 and ASO2 at $15 \mathrm{mpk}$ significantly reduced SCD1 mRNA levels in BAT after 4 and 10 weeks of treatment (Table 3). While ASO2 treatment significantly increased UCP2 mRNA levels only after 4 weeks of treatment, it significantly increased the mRNA levels of not only UCP2 but also UCP1, UCP3, and $\beta 3-A R$ after 10 weeks of treatment (Table 3). ASO2 treatment had no significant effects on VLCAD mRNA levels, which were measured as a control, at either week 4 or week 10 of the treatment in BAT (data not shown).

\section{Discussion}

We have evaluated the potential metabolic effects of pharmacological inhibition of SCD1 in mice. The results show that SCD1 ASOs (ASO1 and ASO2) but not the ASOctrl significantly reduce SCD1 mRNA and protein levels in a dose-dependent fashion, with an $\mathrm{IC}_{50}$ of approximately $150 \mathrm{nM}$ in isolated mouse primary hepatocytes (Figure 1, A-C). This effect was specific for the SCD1 gene, since 


\section{Table 1}

Sequence of TaqMan primers and probes

\begin{tabular}{|c|c|}
\hline Gene & Sequence of primers and probe \\
\hline \multirow[t]{3}{*}{ ACC1 } & F: CCCGCCAGCTTAAGGACA \\
\hline & R: TGGATGGGATGTGGGCA \\
\hline & P: CACCTGTGTGGTGGAATTTCAGTTCATGC \\
\hline \multirow[t]{3}{*}{ ACC2 } & F: ACAGAGATTTCACCGTTGCGT \\
\hline & R: CGCAGCGATGCCATTGT \\
\hline & P: ACTCGCTTTGGAGGCAACAGGGTCAT \\
\hline \multirow[t]{3}{*}{ ACO } & F: GAGTGAGCTGCCTGAGCTTCA \\
\hline & R: TCTTCGATACCAGCATTGGCT \\
\hline & P: AGCTGGGCTGAAGGCTTTTACTACCTGGA \\
\hline \multirow{3}{*}{ L-CPT1 } & F: AATATGTCTACCTCCGAAGCAGGA \\
\hline & R: CGTGAACGGCATTGCCTAG \\
\hline & P: CAACTATTATGCCATGGATTTTGTGCTTATTAAGA \\
\hline \multirow[t]{3}{*}{ FAS } & F: ACCATGGAGCGTATATGTGAACA \\
\hline & R: CAATGCCCACGTCACCAA \\
\hline & P: TGTCCTCCCAGGCCTTGCCGT \\
\hline \multirow{3}{*}{ SCD1 } & F: TTCCGCCACTCGCCTACA \\
\hline & R: CTTTCCCAGTGCTGAGATCGA \\
\hline & P: CAACGGGCTCCGGAACCGAA \\
\hline \multirow[t]{3}{*}{ SREBP-1 } & F: CATCGACTACATCCGCTTCTTG \\
\hline & R: TTTTGTGTGCACTTCGTAGGGT \\
\hline & P: AGCAACCAGAAGCTCAAGCAGGAGAACCT \\
\hline \multirow[t]{3}{*}{ UCP1 } & F: CCTTCCCGCTGGACACTG \\
\hline & R: CCTAGGACACCTTTATACCTAATGGT \\
\hline & P: CAAAGTCCGCCTTCAGATCCAAGGTG \\
\hline \multirow[t]{3}{*}{ UCP2 } & F: CCTCTACGACTCTGTCAAACAGTTCTA \\
\hline & R: GCCAGGGCACCTGTGGT \\
\hline & P: TGCCTGCATGCTCTGAGCCCTTG \\
\hline \multirow[t]{3}{*}{ UCP3 } & F: CCTCTACGACTCTGTTAAGCAGTTCTAC \\
\hline & R: GCTCCTGTCGTGCAGCCT \\
\hline & P: CGCTGGAGTGGTCCGCTCCCTT \\
\hline \multirow[t]{3}{*}{ VLCAD } & F: GCTGAGACGGAGGACAGGAAT \\
\hline & R: ACCACGGTGGCAAATTGATC \\
\hline & P: AGAGCCTGCACTGCCAGTTCACCACT \\
\hline \multirow[t]{3}{*}{$\beta 3-A R$} & F: GGGAGGCAACCTGCTGGTA \\
\hline & R: GAAGTCACGAACACGTTGGTTATG \\
\hline & P: CGCCCGCACGCCGAGACT \\
\hline
\end{tabular}

$\mathrm{F}$, forward; $\mathrm{R}$, reverse; $\mathrm{P}$, probe.

mRNA levels of other genes including $F A S$ were not affected in this in vitro setting. SCD1 ASOs also reduced SCD mRNA levels and/or enzyme activities in liver, WAT, and BAT of C57/B6 mice. SCD1 ASOs also reduced lipid desaturation index in liver and in plasma after in vivo treatment (Figure 4, A and B, Figure 5, and Tables 2 and 3 ). The same ASOs did not affect the mRNA levels of several other genes tested (i.e., VLCAD and $A C O$ ) in the same tissue samples. Finally, no significant metabolic differences were observed between ASOctrl- and saline-treated animals (data not shown). These results demonstrate that SCD1 ASOs reduce the expression and activity of SCD1 in vitro and in multiple insulin responsive tissues in vivo in specific, concentration-dependent, and time-dependent fashion.

We performed in vivo experiments in which C57/B6 mice, who had been on normal chow since weaning, were switched to an HFD; from that time on, mice were treated twice a week with 1 of the 2 SCD1 ASOs or the ASOctrl for 10 weeks at 5 and/or $15 \mathrm{mpk}$. Mice treated with either SCD1 ASO at 15 but not at $5 \mathrm{mpk}$ showed significantly reduced body weight gain mainly due to reduced body adiposity (Figure 2, $\mathrm{A}$ and $\mathrm{C}$ ). These results correlate with the phenotypes of SCD1-deficient mice $(12,15,17,22)$. However, since mice treated with SCD1 ASOs do not have apparent hair loss and skin abnormalities (data not shown), the current study demonstrates, for the first time to our knowledge, that reduction in body weight and adiposity in mice deficient in or with reduced SCD1 can occur in the absence of hair and skin lesions. It is important to note that, although the lack of hair loss does not formally exclude the possibility that the ASOs may nevertheless reduce SCD1 activity in skin and alter sebaceous gland histology and function, such effect on skin is unlikely to occur, since ASOs are documented as having limited tissue distribution and preferentially target the liver.

Although it was reported that SCD1-deficient mice are somewhat hyperphagic (12), food intake by mice treated with SCD1 ASOs was similar to that of mice treated with ASOctrl (Figure 2B). This suggests that the reduction of body weight and adiposity in mice treated with SCD1 ASOs was not mainly due to changes in food intake. It was reported that $S C D 1$-deficient mice have increased energy expenditure $(12,17)$. We observed in the current study significant increases in oxygen consumption by mice treated with ASO2 at $15 \mathrm{mpk}$ (Figure 3A). The interpretation of the increased oxygen consumption, however, is somewhat complicated by the observation that the SCD1 ASO-treated mice also exhibited increased physical activity (Figure 3B). Since physical activity was measured at week 10 when the ASO2-treated animals were significantly leaner (Figure 2A), it is possible that the increased physical activity in these mice may be a consequence of their leanness. We do not exclude the possibility that increased physical activity may contribute to the leanness in the ASO2-treated mice at least at the later stage of the experiment. However, it is likely that the observed increase in resting metabolic rate in ASO2-treated mice, which confirms earlier findings from studies on $S C D 1^{-/}$mice (20), is responsible, at least in part, for the resistance to diet-induced obesity in ASO2-treated mice. The concept of augmented energy expenditure in ASO2-treated mice is supported in the current study by the increased expression of UCP proteins (UCP1-3) as well as $\beta 3$-ARs in BAT (Table 3). Furthermore, SCD1 ASOs increased fatty acid oxidization in primary mouse hepatocytes in vitro (Figure $1 \mathrm{H}$ ) and increased the hepatic expression of $L$-CPT1, a key regulator of fatty acid oxidation (Table 1). The changes in the levels of expression of these genes in concert could result in increased energy expenditure in multiple insulin-responsive tissues, which could at least be partially responsible for SCD1 ASO-mediated protection against diet-induced adiposity in mice.

In addition to increased energy expenditure, reduced de novo fatty acid synthesis also contributes to SDC1 ASO-mediated reduction in weight gain and body adiposity. SCD1 ASOs decreased fatty acid but not sterol synthesis and secretion by primary mouse hepatocytes in vitro (Figure 1, D-G). The treatments significantly decreased de novo fatty acid synthesis activity (Figure 4, C and D) and steatosis (Figure 4, E-H) in the liver of treated mice. Gene expression analyses showed that SCD1 ASO treatment reduced the expression of multiple lipogenic genes (i.e., FAS, ACC1, ACC2, and SREBP-1) in liver (Table 2) and the expression of FAS in WAT (Table 3 ) in a time- and dose-dependent fashion. Downregulation of FAS and SREBP-1 in the livers of SCD1-deficient mice has also been reported (17). In contrast to what was observed in SCD1-deficient mice, there were no significant changes in the expression of $A C O, V L C A D$, and fasting-induced adipocyte factor (FIAF) in the livers of SCD1 ASO-treated mice (data not shown). It has been reported that ASOs administered i.p. can reduce target genes in adipose tissue (23-25). Nevertheless, it is possible that the inhibition of SCD1 


\section{Table 2}

Levels of mRNA of genes in the livers of mice treated with AS01 and ASO2 relative to those treated with $\mathrm{ASO} \mathrm{ctrl}(\%)^{\mathrm{A}}$

\begin{tabular}{lcccccc} 
& \multicolumn{2}{c}{ Week 4 } & \multicolumn{4}{c}{ Week 10 } \\
Gene & AS01, & AS02, & AS01, & AS01, & AS02, & AS02, \\
& $\mathbf{1 5} \mathbf{~ m p k}$ & $\mathbf{1 5} \mathbf{~ m p k}$ & $\mathbf{5 ~} \mathbf{~ p k}$ & $\mathbf{1 5} \mathbf{~ m p k}$ & $\mathbf{5 ~ m p k}$ & $\mathbf{1 5} \mathbf{~ m p k}$ \\
SCD1 & $49 \pm 7^{\mathrm{B}}$ & $53 \pm 12^{\mathrm{B}}$ & $58 \pm 16$ & $41 \pm 9^{\mathrm{C}}$ & $50 \pm 15^{\mathrm{C}}$ & $37 \pm 5^{\mathrm{C}}$ \\
FAS & $78 \pm 5$ & $82 \pm 18$ & $58 \pm 15$ & $41 \pm 9^{\mathrm{C}}$ & $50 \pm 13$ & $37 \pm 5^{\mathrm{C}}$ \\
L-CPT1 $^{3}$ & $390 \pm 90^{\mathrm{B}}$ & $202 \pm 35^{\mathrm{C}}$ & $143 \pm 20$ & $215 \pm 16^{\mathrm{B}}$ & $108 \pm 12$ & $175 \pm 18^{\mathrm{C}}$ \\
ACC1 & $72 \pm 10$ & $118 \pm 17$ & $65 \pm 6^{\mathrm{B}}$ & $69 \pm 8^{\mathrm{B}}$ & $74 \pm 8^{\mathrm{C}}$ & $72 \pm 8^{\mathrm{C}}$ \\
ACC2 & $46 \pm 7^{\mathrm{C}}$ & $51 \pm 9^{\mathrm{C}}$ & $58 \pm 15$ & $41 \pm 9^{\mathrm{C}}$ & $40 \pm 8^{\mathrm{C}}$ & $37 \pm 5^{\mathrm{C}}$ \\
SREBP & $66 \pm 5^{\mathrm{C}}$ & $83 \pm 10$ & $77 \pm 12$ & $59 \pm 6^{\mathrm{B}}$ & $67 \pm 11^{\mathrm{C}}$ & $53 \pm 6^{\mathrm{B}}$
\end{tabular}

AThe levels of mRNA were determined by TaqMan real-time PCR. The level of mRNA of each of the genes was set to $100 \%$ in liver tissues treated with ASOctrl at $15 \mathrm{mpk}$ in weeks 4 and 10. ${ }^{B} P<0.01,{ }^{C} P<0.05$; Student's $t$ test.

by ASO treatment in the adipose tissues could be a secondary effect of reducing SCD1 expression in the liver.

In comparison with wild type mice, SCD1-knockout mice have lower fasting insulin levels on chow diet but not on HFD. While the fasting glucose is comparable between the wild-type and the SCD1-knockout mice, the latter have improved glucose tolerance (17). Furthermore, it was reported that decreased expression and activity of protein tyrosine phosphatase PTP1B in the muscle may play a role in improving insulin sensitivity in SCD1-deficient mice by potentiating the insulin-signaling cascade and glucose transporter 4 (GLUT4) translocation to cell surface (22). On the other hand, aP2-nSREBP-1c mice that are also homozygous for SCD1 deficiency do not have improved insulin sensitivity when compared with aP2-nSREBP-1c mice. Furthermore, without affecting hepatic steatosis and SCD1 activity, subcutaneous leptin at low dosage potentiates insulin signaling in aP2-nSREBP-1c mice (18). The role of SCD1 in insulin sensitivity therefore remains somewhat unclear. Interestingly, the current study showed that SCD1 ASO treatment reduced the postprandial plasma insulin and glucose levels in the C57/B6 mice (Figure 2, D and E), which supports the notion that SCD1 deficiency improves insulin sensitivity in mice. While the current study did not investigate the effect of SCD1 ASO treatment on gene expression in muscle, PTP1B mRNA levels in both the livers and the WAT in the C57/B6 mice were not significantly altered (data not shown).

Treatment with SCD1 ASOs at $5 \mathrm{mpk}$ did not affect body weight (Figure 3A), body composition (data not shown), and blood glucose (data not shown). Such treatments nevertheless significantly reduced SCD1 mRNA levels by approximately 50\% in liver (Table 2). This suggests that greater than 50\% inhibition of SCD1 activity is required to bring about discernible metabolic effects. Furthermore, these results indicate that the metabolic effects of SCD1 ASOs are not merely mediated by the direct inhibition of SCD1 expression and activity per se. Rather, SCD1 inhibition leads to a broader reprogramming of pathways regulating lipid metabolism, and it is such reprogramming that brings about the metabolic phenotypes. The proposed molecular basis for SCD1 ASO-mediated metabolic effects is shown schematically in Figure 6.

In summary, the current study demonstrates that pharmacological inhibition of SCD1 protects mice from diet-induced obesity and improves insulin sensitivity in the absence of alopecia. The metabolic effects are mediated by synergistic effects of reduced fatty acid synthesis and increased energy expenditure, which are consistent with a coordinated regulation of relevant genes important in lipid metabolism and energy expenditure in multiple insulin-responsive tissues (liver, WAT, and BAT). Thus, SCD1 inhibitors hold potential for the treatment of obesity and metabolic syndrome.

\section{Methods}

SCD1 ASOs and ASOctrl. Among 78 ASOs designed against mouse SCD1 mRNA, SCD1 ASO1 (5'-gtgttTCTGAGAACTtgtgg-3') was selected based on its ability to inhibit SCD1 mRNA levels in primary mouse hepatocytes. A second SCD1 ASO (ASO2) is identical to ASO1 in sequence but is a gap-ablated version ( $5^{\prime}$-gtgTTtCTgAGaACtTGtgg- $\left.3^{\prime}\right)$. Finally, a control ASO (ASOctrl) with a random sequence $\left(5^{\prime}\right.$-ccttcCCTGAAGGTTcctcc- $\left.3^{\prime}\right)$ was also prepared. The ASOs were prepared as described previously (26).

Preparation of animals. C57/B6 mice were purchased from Taconic. Prior to this study, mice were group housed and provided with ad libitum rodent chow (Teklad 7012; Harlan Teklad Global Diets) and reverse osmosis water via a water bottle. The light-dark cycle was 7:00 am-7:00 pm, and the building was provided with high-efficiency particulate air-filtered (HEPAfiltered) air, maintained at $72 \pm 2^{\circ} \mathrm{F}$. All animal procedures involved the humane care and use of animals, were performed within an Association for Assessment and Accreditation of Laboratory Animal Care Internationalaccredited facility, and were approved by the Merck-Rahway Institutional Animal Care and Use Committee.

Isolation and transfection of primary hepatocytes from C57/B6 mice. Hepatocytes were isolated from the C57/B6 mice as previously described (27). Briefly, livers in anesthetized mice were perfused with freshly pre-
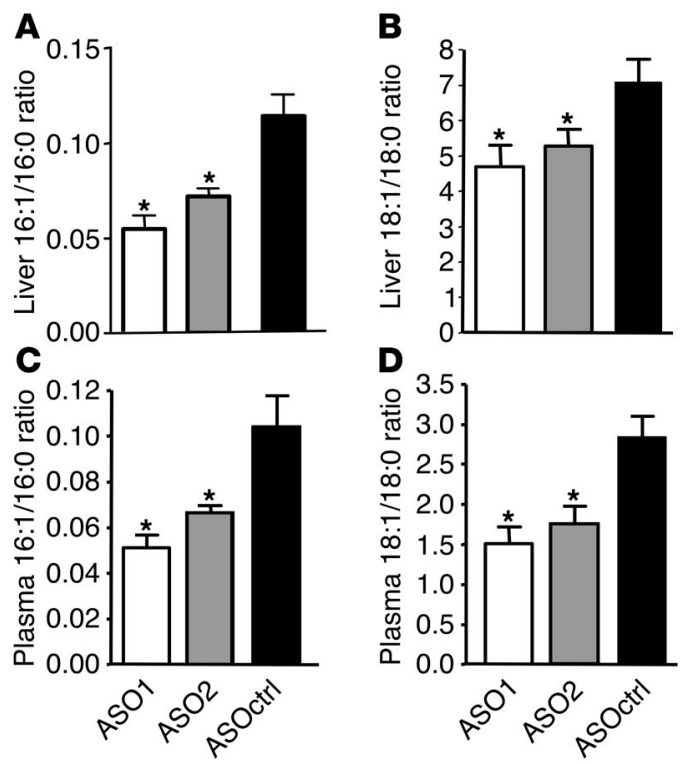

\section{Figure 5}

Effect of SCD1 ASOs on lipid desaturation index in liver and plasma of treated C57/B6 mice on HFD. Saturated fatty acids and MUFAs in liver and plasma were measured after mice were treated with the various ASOs at $15 \mathrm{mpk}$ for 4 weeks as described in Methods. Shown are the ratios of the levels of MUFAs verses saturated fatty acids in liver (A and $\mathbf{B}$ ) and in plasma (C and $\mathbf{D})$. 


\section{Table 3}

Levels of mRNA of genes in the WAT and BAT of mice treated with ASO2 relative to those treated with ASOctrl $(\%)^{A}$

\begin{tabular}{lrrrr} 
& \multicolumn{2}{c}{ WAT } & \multicolumn{2}{c}{ BAT } \\
Gene & AS02, & AS02, & AS02, & AS02, \\
& $\mathbf{1 5} \mathbf{~ m p k ,}$ & $\mathbf{1 5} \mathbf{~ m p k ,}$ & $\mathbf{1 5} \mathbf{~ m p k ,}$ & $\mathbf{1 5} \mathbf{~ m p k}$, \\
& week 4 & week 10 & week 4 & week 10 \\
SCD1 & $33 \pm 9 \mathrm{~B}$ & $32 \pm 7^{\mathrm{C}}$ & $20 \pm 3^{\mathrm{B}}$ & $59 \pm 11^{\mathrm{B}}$ \\
FAS & $85 \pm 31$ & $25 \pm 4 \mathrm{C}$ & & \\
UCP1 & & & $96 \pm 15$ & $138 \pm 8^{\mathrm{C}}$ \\
UCP2 & & & $164 \pm 11^{\mathrm{C}}$ & $139 \pm 12^{\mathrm{B}}$ \\
UCP3 & & & $120 \pm 10$ & $144 \pm 17^{\mathrm{B}}$ \\
B3-AR & & & $97 \pm 10$ & $145 \pm 8^{\mathrm{C}}$
\end{tabular}

AThe levels of mRNA were determined by TaqMan real-time PCR. The level of mRNA of each of the genes was set to $100 \%$ in WAT or BAT treated with ASOctrl at 15 mpk in weeks 4 and $10 .{ }^{B} P<0.05,{ }^{C} P<0.01$; Student's $t$ test.

pared Leffert's buffer (10 mM HEPES, $3 \mathrm{mM} \mathrm{KCl}, 130 \mathrm{mM} \mathrm{NaCl}, 1 \mathrm{mM}$ $\mathrm{NaH}_{2} \mathrm{PO} 4,10 \mathrm{mM}$ D-glucose, $\mathrm{pH}$ 7.4) with $0.5 \mathrm{mM}$ EGTA via the portal vein for 5 minutes and then with digestion buffer (Leffert's buffer plus collagenase I from Worthington Biochemical Corp. at $0.33 \mathrm{mg} / \mathrm{ml}$ and $0.0279 \% \mathrm{CaCl}_{2}$ ) for 12 minutes. The perfused livers were removed from the mice and dissociated in ice-cold wash buffer (Leffert's buffer plus $0.0279 \%$ $\mathrm{CaCl}_{2}$ ) by agitation. The hepatocytes were washed with ice-cold wash buffer 3 times by centrifugation; seeded onto Primaria tissue-culture plates (BD Biosciences) at 400,000 to 600,000 cells per well in $2 \mathrm{ml}$ hepatocyte culture medium (RPMI plus 10\% FCS, $100 \mathrm{nM}$ dexamethasone, $10 \mu \mathrm{g} / \mathrm{ml}$ insulin, and $5 \mu \mathrm{g} / \mathrm{ml}$ transferrin); and cultured overnight at $37^{\circ} \mathrm{C}$ and $5 \% \mathrm{CO}_{2}$. For transfection, hepatocytes in the 6-well plates were washed gently once with PBS and then incubated with $1 \mathrm{ml}$ of transfection mixture per well for 4-6 hours. The transfection mixture contained $2.5 \mu \mathrm{L}$ Lipofectin $/ \mathrm{ml}$ (Invitrogen Corp.) per $100 \mathrm{nM}$ ASO (i.e., $20 \mu \mathrm{l}$ Lipofectin in each milliliter of mixture for transfection with $800 \mathrm{nM}$ of ASO). The transfection mix was then aspirated and replaced with $3 \mathrm{ml}$ hepatocyte culture medium. The transfected cells were allowed to recover overnight for subsequent RNA extraction, protein extraction, and/or metabolic studies.

Determination of fatty acid and sterol synthesis, secretion, and fatty acid oxidation in transfected hepatocytes in vitro. Fatty acids and sterol synthesis in the transfected primary hepatocytes were measured for the most part as previously described $(28,29)$ with some modifications. Briefly, after overnight culture, transfected hepatocytes were washed twice with PBS and then incubated with $2 \mathrm{ml}$ highglucose DMEM plus $\left[{ }^{14} \mathrm{C}\right]$ acetate (New England Nuclear) at $0.5 \mu \mathrm{Ci}$ per well. The wells were gassed with $95 \% \mathrm{O}_{2}$ and $5 \% \mathrm{CO}_{2}$, and the plates were sealed with Parafilm. After overnight ( 17 hours) incubation, tissue culture medium $(2 \mathrm{ml})$ or the cells washed twice with PBS were mixed with $2.5 \mathrm{ml} \mathrm{10 \%} \mathrm{KOH}$ in methanol and $1.0 \mathrm{ml}$ distilled water per well. The mixture was heated at $90^{\circ} \mathrm{C}$ for 3 hours and extracted with $4 \mathrm{ml}$ petroleum ether $(\mathrm{PE})$ once. Three milliliters of the upper PE layer was transferred into a scintillation vial and dried under low heat, and then the number of $\left[{ }^{14} \mathrm{C}\right]$ sterols was determined. Three milliliters of the lower aqueous layer was mixed first with $1.0 \mathrm{ml}$ of $10 \mathrm{M} \mathrm{H}_{2} \mathrm{SO}_{4}$ and then with $4 \mathrm{ml}$ of PE. The mixture was then subject to centrifugation, after which $3 \mathrm{ml}$ of the upper layer was obtained and dried under low heat, and the levels of $\left[{ }^{14} \mathrm{C}\right]$-labeled fatty acids were determined.

We determined fatty acid oxidation in the transfected primary hepatocytes by measuring the oxidation of $\left[{ }^{14} \mathrm{C}\right]$ oleate to acid soluble products for the most part as previously described (30) with some modifications. Briefly, hepatocytes were washed with Medium 199 (HyClone) and then incubated with $2 \mathrm{ml}$ Medium 199 plus $0.25 \%$ BSA and $0.25 \mu \mathrm{Ci}$ $\left[{ }^{14} \mathrm{C}\right]$ oleic acid (New England Nuclear). The wells were gassed with 95\% $\mathrm{O}_{2}$ and $5 \% \mathrm{CO}_{2}$, and the plates were sealed with Parafilm. After overnight (17 hours) incubation, $1 \mathrm{ml}$ of medium was removed and mixed with $100 \mu \mathrm{l}$ of $10 \% \mathrm{BSA}$ and then $100 \mathrm{ml} 60 \% \mathrm{HClO}_{4}$. After centrifugation, the level of soluble $\left[{ }^{14} \mathrm{C}\right]$-labeled products in $1 \mathrm{ml}$ of the solution was determined.

In vivo experiments with $\mathrm{C57/B6}$ mice. $\mathrm{C} 57 / \mathrm{B} 6$ mice fed a chow diet were approximately 5 weeks old at the beginning of the experiments. Mice were switched from a standard rodent chow to HFD D12492 containing 60\% $\mathrm{kCal}$ of mostly saturated fats (Research Diets Inc.) and treated with ASOs dissolved in saline at 5 and $15 \mathrm{mpk}$ twice a week via i.p. injection for 10 weeks. We chose to treat animals twice a week because, based on our previous experience with other ASOs of similar chemistry, such a dosing scheme provides sustained coverage (data not shown). A 10-week study was performed to determine whether any metabolic effects exerted by SCD1 ASOs would be sustainable over time. There were 16-24 animals per treatment group. Body weight and food intake were monitored weekly. Blood was obtained in the postprandial state by tail vein bleeding at weeks 4 and 10 for measurement of insulin, glucose, free fatty acids, triglycerides, and glucagon concentrations, as previously described (31). Body composition was assessed by a Fat/Lean Mice Whole Body Magnetic Resonance Analyzer (Bruker). Liver tissue, WAT, and BAT from animals treated for 4 and 10 weeks were obtained for RNA isolation and TaqMan analysis of gene expression.

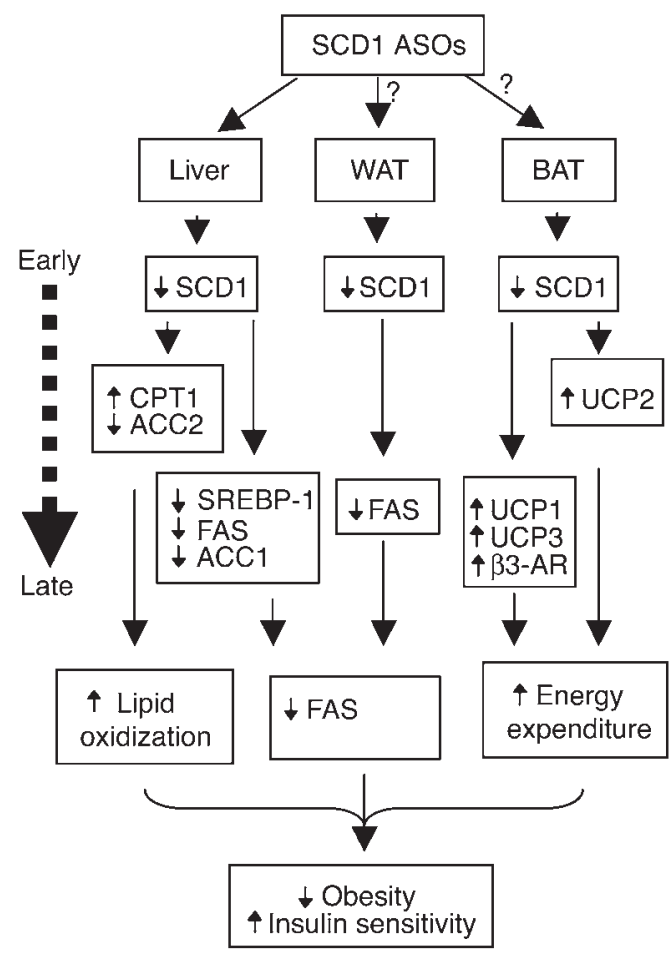

Figure 6

Proposed molecular basis for SCD1 ASO-mediated metabolic effects in mice. SCD1 ASOs directly reduce SCD1 levels in liver. SCD1 ASOs also reduce SCD1 in WAT and BAT; however, whether the reduction is due to direct or indirect effects remains unknown and is indicated by question marks. In liver, the reduction of SCD1 leads to increased CPT1 levels first and decreased SREBP-1, FAS, ACC1, and ACC2 later. It is possible that the downregulation of FAS, ACC1, and ACC2 may be downstream of the transcription factor SREBP-1. In WAT, reduction of SCD1 leads to reduction of FAS. In BAT, reduction of SCD1 leads to increase in UCP2 first and then UCP1, UCP3, and $\beta 3-A R$. 
De novo whole body fatty acid and sterol synthesis in liver in vivo were measured as previously described (32) with some modifications. Briefly, mice were treated with $0.5 \mathrm{ml}$ of $0.15 \mathrm{~N} \mathrm{NaCl}$ containing $0.2 \mathrm{mCi}$ of $\left[{ }^{3} \mathrm{H}\right]$ water/100 g body weight by i.p. injection. Liver samples (approximately $700 \mathrm{mg}$ per animal) were excised from the animals 1 hour later, heated at $90^{\circ} \mathrm{C}$ for 5 hours in a mixture of $1.5 \mathrm{ml} 4 \mathrm{M} \mathrm{KOH}$ and $1.5 \mathrm{ml}$ of $95 \%$ ethanol. The alkaline hydrolysate was then mixed with $4 \mathrm{ml}$ hexane. After centrifugation, the organic phase was obtained and dried under warm air, and then the level of $\left[{ }^{3} \mathrm{H}\right]$ sterols was determined. The aqueous phase $(3 \mathrm{ml})$ was acidified with $0.75 \mathrm{ml}$ of $10 \mathrm{M} \mathrm{H}_{2} \mathrm{SO}_{4}$, mixed with $4 \mathrm{ml}$ hexane, and subjected to centrifugation. The organic phase was then backwashed with $3 \mathrm{ml}$ of distilled water, then dried and counted to determine the levels of $\left[{ }^{3} \mathrm{H}\right]$-labeled fatty acids.

Determination of energy expenditure and physical activity. We quantified energy expenditure by measuring oxygen consumption in an open-air circuit indirect calorimetry system (Oxymax Equal Flow; Columbus Instruments). Briefly, mice were placed in calorimetric chambers at $24^{\circ} \mathrm{C}$ with free access to food and water for a period of approximately 24 hours. The light-dark cycle was 7:00 am-7:00 pm. Expired gases from each chamber were collected and analyzed for $\mathrm{CO}_{2}$ and $\mathrm{O}_{2}$ content every 3 minutes. Room air was sampled every 27 minutes as a reference, and air flow through the chambers was $600 \mathrm{ml} / \mathrm{min}$. Oxygen consumption was expressed as $\mathrm{ml} \mathrm{O}_{2} / \mathrm{kg}$ body $\mathrm{wt} / \mathrm{h}$. Physical activity was determined simultaneously with the indirect calorimetry studies. Individual calorimetric chambers were equipped with infrared beams in the $x y z$ axis. Data reported represent cumulative ambulatory movements, which mostly exclude movement typical of grooming and are reflective of locomotor activity.

RNA isolation and quantification of gene expression. Cultured primary hepatocytes or frozen tissue samples (approximately 50-200 mg) were lysed in $1 \mathrm{ml}$ of Ultraspec Total RNA Isolation Reagent (Biotecx Laboratories Inc.). The lysates were mixed with $200 \mu \mathrm{l}$ chloroform and then subjected to centrifugation. The aqueous phase was mixed with 0.6 volume of $100 \%$ ethanol and then passed through an RNeasy 96 Plate (QIAGEN). RNase-Free DNase I (QIAGEN) in RDD buffer $(80 \mu \mathrm{l})$ was added to each of the wells. After 5 minutes incubation at room temperature, the plate was centrifuged, washed subsequently with buffer RW1 and RPE, and eluted with $50 \mu \mathrm{l}$ of RNase-free water. For RNA from animal tissues, we subjected the RNA eluted from the RNeasy 96 Plate to further purification to remove contaminating ASO using the QIAquick PCR Purification Kit (QIAGEN). Briefly, the RNA was mixed with $5 \times$ volume of binding buffer, passed via the column by centrifugation, washed once with wash buffer, and eluted with $50 \mu \mathrm{l}$ RNase-free water. We performed the synthesis of cDNA from RNA and the quantitative amplification of target cDNAs by TaqMan PCR using reagent kits in the ABI PRISM 7700 sequence detection system per the manufacturer's instructions (Applied Biosystems). The TaqMan primer/probe sets used are shown in Table 1. The level of mRNA for each gene was normalized to that of $18 \mathrm{~S}$ ribosomal RNA in the same sample.

Protein extraction and Western blot analysis. Proteins from primary mouse hepatocytes were extracted, and Western blot analysis was performed as previously described (31) using the polyclonal antibody against murine SCD1 from Santa Cruz Biotechnology Inc.

Determination of SCD enzyme activity. $\left[{ }^{14} \mathrm{C}\right]$ stearoyl-CoA was purchased from American Radiolabeled Chemicals Inc. We measured SCD activity in liver microsomes by monitoring the conversion of $\left[{ }^{14} \mathrm{C}\right]$ stearoyl-CoA to $\left[{ }^{14} \mathrm{C}\right]$ oleoyl-CoA. Briefly, tissues were homogenized in 10 volumes buffer (25 mM sucrose, $1 \mathrm{mM}$ EDTA, $5 \mathrm{mM}$ DTT, $50 \mathrm{mM}$ Tris-HCl, $\mathrm{pH}$ 7.4). The microsomal membrane fraction $(100,000 \mathrm{~g}$ pellet) was isolated by sequential centrifugation and resuspended in buffer containing $20 \%$ glycerol, $2 \mathrm{mM}$ DTT, $100 \mathrm{mM}$ sodium phosphate, $\mathrm{pH}$ 7.4. The assay system contained $2 \mathrm{mM}$ NADH, $2 \mathrm{mM}$ DTT, $0.06 \mathrm{mM}\left[{ }^{14} \mathrm{C}\right]$ stearoyl-CoA (specific activity $50 \mathrm{mCi} / \mathrm{mmol}$ ), $100 \mathrm{mM}$ Tris, $\mathrm{pH} 7.2$, and liver microsomes in a final volume of $0.1 \mathrm{ml}$. The reaction was initiated by the addition of microsomes and incubated at $37^{\circ} \mathrm{C}$ for 3 minutes. The reaction was halted by the addition of $0.225 \mathrm{ml} 6 \mathrm{mM} \mathrm{H}_{3} \mathrm{PO}_{4}$ in ethanol. Samples were centrifuged for 5 minutes and the supernatant applied to a Betasil C-18 column (Thermo Electron Corp.) equilibrated at $1 \mathrm{ml} / \mathrm{min}$ in $20 \%$ acetonitrile, $80 \% 10 \mathrm{mM}$ $\mathrm{KH}_{2} \mathrm{PO}_{4}$, pH 5.3, 70\% acetonitrile, $30 \% 10 \mathrm{mM} \mathrm{KH}_{2} \mathrm{PO}_{4}$, pH 5.3 (42:58). The conversion of $\left[{ }^{14} \mathrm{C}\right]$ stearoyl-CoA to $\left[{ }^{14} \mathrm{C}\right]$ oleoyl-CoA was monitored via a Packard 500TR Series Flow Scintillation Analyzer.

Histological analysis. Liver tissue samples were fixed in $10 \%$ buffered neutral formalin. Half of the sample was processed and sectioned in paraffin and half was frozen and sectioned on a cryostat. Paraffin sections were stained with H\&E to visualize liver histology. Frozen sections were stained with oil red $\mathrm{O}$ to visualize fat deposits in the liver.

Measurement of desaturation index. Lipids were extracted from plasma or liver as previously described (33). Briefly, fatty acid methyl esters were prepared, extracted, dried down, and resuspended in hexane. Fatty acid methyl esters were identified with an Agilent 6890 gas chromatograph connected to an HP- 5 column $(30 \mathrm{~m} \times 0.32 \mathrm{~mm} \times 0.25 \mu \mathrm{M}$ film thickness $)$ connected to a flame ionization detector set at $2,500^{\circ} \mathrm{C}$. The column and the injector temperature were set to $500^{\circ} \mathrm{C}$. The column temperature was increased to $1,500^{\circ} \mathrm{C}$ at $40^{\circ} \mathrm{C} / \mathrm{min}$, increased to $2,000^{\circ} \mathrm{C}$ at $30^{\circ} \mathrm{C} / \mathrm{min}$, held at $2,000^{\circ} \mathrm{C}$ for 25 minutes, and then increased to $2,250^{\circ} \mathrm{C}$ at $250^{\circ} \mathrm{C} / \mathrm{min}$ and held at $2,250^{\circ} \mathrm{C}$ for 10 minutes. Under these conditions, the $\Delta 9-16: 1$, 16:0, $\Delta 9-18: 1$, and 18:0 methyl esters eluted at 14.2, 14.8, 19.4, and 20.2 minutes, respectively. The desaturation index was calculated as the ratios of $\Delta 9-16: 1 / 16: 0$ and $\Delta 9-18: 1 / 18: 0$.

Statistical analysis. Comparisons among treatments were performed using nonpaired Student's $t$ test. $P$ values less than 0.05 were considered significant.

Received for publication November 22, 2004, and accepted in revised form January 25, 2005.

Address correspondence to: Bei B.Zhang, RY80W-180, Merck Research Laboratories, PO Box 2000, Rahway, New Jersey 07065, USA. E-mail: bei_zhang@merck.com.

Guoquiang Jiang and Zhihua Li contributed equally to this work.
1. Kopelman, P.G. 2000. Obesity as a medical problem. Nature. 404:635-643.

2. Abelson, P., and Kennedy, D. 2004. The obesity epidemic. Science. 304:1413.

3. Enoch, H.G., Catala, A., and Strittmatter, P. 1976. Mechanism of rat liver microsomal stearyl-CoA desaturase. Studies of the substrate specificity, enzyme-substrate interactions, and the function of lipid. J. Biol. Chem. 251:5095-5103.

4. Ntambi, J.M., and Miyazaki, M. 2004. Regulation of stearoyl-CoA desaturases and role in metabolism. Prog. Lipid Res. 43:91-104.

5. Zheng, Y., et al. 2001. Scd3--a novel gene of the
stearoyl-CoA desaturase family with restricted expression in skin. Genomics. 71:182-191.

6. Miyazaki, M., et al. 2003. Identification and characterization of murine SCD4, a novel heart-specific stearoyl-CoA desaturase isoform regulated by leptin and dietary factors. J. Biol. Chem. 278:33904-33911.

7. Zhang, L., Ge, L., Parimoo, S., Stenn, K., and Prouty, S.M. 1999. Human stearoyl-CoA desaturase: alternative transcripts generated from a single gene by usage of tandem polyadenylation sites. Biochem. J. 340:255-264.

8. Beiraghi, S., et al. 2003. Identification and characterization of a novel gene disrupted by a pericentric inversion inv(4)(p13.1q21.1) in a family with cleft lip. Gene. 309:11-21.

9. Ntambi, J.M. 1995. The regulation of stearoyl-CoA desaturase (SCD). Prog. Lipid. Res. 34:139-150.

10. Waters, K.M., and Ntambi, J.M. 1994. Insulin and dietary fructose induce stearoyl-CoA desaturase 1 gene expression of diabetic mice. J. Biol. Chem. 269:27773-27777.

11. Ntambi, J.M. 1992. Dietary regulation of stearoylCoA desaturase 1 gene expression in mouse liver. J. Biol. Chem. 267:10925-10930.

12. Cohen, P., Ntambi, J.M., and Friedman, J.M. 2003. Stearoyl-CoA desaturase- 1 and the metabolic syn- 
drome. Curr. Drug Targets Immune Endocr. Metabol. Disord. 3:271-280.

13. Dobrzyn, A., and Ntambi, J.M. 2004. The role of stearoyl-CoA desaturase in body weight regulation. Trends Cardiovasc. Med. 14:77-81.

14. Zheng, Y., et al. 1999. Scd1 is expressed in sebaceous glands and is disrupted in the asebia mouse. Nat. Genet. 23:268-270.

15. Miyazaki, M., Kim, Y.C., Gray-Keller, M.P., Attie, A.D., and Ntambi, J.M. 2000. The biosynthesis of hepatic cholesterol esters and triglycerides is impaired in mice with a disruption of the gene for stearoyl-CoA desaturase 1. J. Biol. Chem. 275:30132-30138.

16. Cohen, P., et al. 2002. Role for stearoyl-CoA desaturase-1 in leptin-mediated weight loss. Science. 297:240-243

17. Ntambi, J.M., et al. 2002. Loss of stearoyl-CoA desaturase- 1 function protects mice against adiposity. Proc. Natl. Acad. Sci. U. S. A. 99:11482-11486.

18. Asilmaz, E., et al. 2004. Site and mechanism of leptin action in a rodent form of congenital lipodystrophy. J. Clin. Invest. 113:414-424. doi:10.1172/JCI200419511.

19. Dobrzyn, P., et al. 2004. Stearoyl-CoA desaturase 1 deficiency increases fatty acid oxidation by activating AMP-activated protein kinase in liver. Proc. Natl. Acad. Sci. U. S. A. 101:6409-6414.
20. Lee, S.H., et al. 2004. Lack of stearoyl-CoA desaturase 1 upregulates basal thermogenesis but causes hypothermia in a cold environment. J. Lipid Res. 45:1674-1682.

21. Attie, A.D., et al. 2002. Relationship between stearoyl-CoA desaturase activity and plasma triglycerides in human and mouse hypertriglyceridemia. J. Lipid Res. 43:1899-1907.

22. Rahman, S.M., et al. 2003. Stearoyl-CoA desaturase 1 deficiency elevates insulin-signaling components and down-regulates protein-tyrosine phosphatase 1B in muscle. Proc. Natl. Acad. Sci. U. S. A. 100:11110-11115.

23. Araújo, E.P., et al. 2005. Short-term in vivo inhibition of insulin receptor substrate- 1 expression leads to insulin resistance, hyperinsulinemia and increased adiposity. Endocrinology. 146:1428-1437.

24. Waring, J.F., et al. 2003. PTP1B antisense-treated mice show regulation of genes involved in lipogenesis in liver and fat. Mol. Cell. Endocrinol. 203:155-168.

25. Muse, E.D., et al. 2004. Role of resistin in dietinduced hepatic insulin resistance. J. Clin. Invest. 114:232-239. doi:10.1172/JCI200421270.

26. Baker, B.F., et al. 1997. 2'-O-(2-Methoxy)ethylmodified anti-intercellular adhesion molecule 1 (ICAM-1) oligonucleotides selectively increase the ICAM-1 mRNA level and inhibit formation of the ICAM-1 translation initiation complex in human umbilical vein endothelial cells. J. Biol. Chem. 272:11994-12000.

27. Neufeld, D.S. 1997. Isolation of rat liver hepatocytes. Methods Mol. Biol. 75:145-151.

28. McGarry, J.D., Takabayashi, Y., and Foster, D.W. 1978. The role of malonyl-coa in the coordination of fatty acid synthesis and oxidation in isolated rat hepatocytes. J. Biol. Chem. 253:8294-8300.

29. Kalopissis, A.D., Griglio, S., Malewiak, M.I., Rozen, R., and Liepvre, X.L. 1981. Very-low-density-lipoprotein secretion by isolated hepatocytes of fat-fed rats. Biochem. J. 198:373-377.

30. Mannaerts, G.P., Debeer, L.J., Thomas, J., and De Schepper, P.J. 1979. Mitochondrial and peroxisomal fatty acid oxidation in liver homogenates and isolated hepatocytes from control and clofibratetreated rats. J. Biol. Chem. 254:4585-4595.

31. Jiang, G., et al. 2002. Potentiation of insulin signaling in tissues of Zucker obese rats after acute and long-term treatment with PPARgamma agonists. Diabetes. 51:2412-2419.

32. Sakurai, T., Miyazawa, S., Shindo, Y., and Hashimoto, T. 1974. The effect of tryptophan administration on fatty acid synthesis in the liver of the fasted normal rat. Biochim. Biophys. Acta. 360:275-288.

33. Dole, V.P. 1956. A relation between non-esterified fatty acids in plasma and the metabolism of glucose. J. Clin. Invest. 35:150-154. 